\title{
Erratum: Multipartite entanglement of fermionic systems in noninertial frames [Phys. Rev. A 83, 022314 (2011)]
}

Jieci Wang and Jiliang Jing

(Received 16 January 2018; published 13 February 2018)

DOI: 10.1103/PhysRevA.97.029902

A few errors were found in Eqs. (15)-(17) and (19) of our article, which are produced by the difference between the square-root order $\sqrt{\rho^{\dagger} \rho}$ in the "Mathematica" program and the square-root $\sqrt{\rho^{\dagger} \rho}$ in quantum theory.

Equations (15)-(17) in the original paper should be

$$
\begin{aligned}
& \mathcal{N}_{A\left(B_{I} C_{I}\right)}=\frac{1}{2}\left(\sqrt{4 \cos ^{2} r_{b} \cos ^{2} r_{c}+\sin ^{4} r_{b} \sin ^{4} r_{c}}-\sin ^{2} r_{c} \sin ^{2} r_{b}\right), \\
& \mathcal{N}_{B_{I}\left(A C_{I}\right)}=\frac{1}{2} \cos r_{c}\left(\sqrt{4 \cos ^{2} r_{b}+\sin ^{4} r_{b} \cos ^{2} r_{c}}-\sin ^{2} r_{b} \cos r_{c}\right),
\end{aligned}
$$

and

$$
\mathcal{N}_{C_{I}\left(A B_{I}\right)}=\frac{1}{2} \cos r_{b}\left(\sqrt{4 \cos ^{2} r_{c}+\sin ^{4} r_{c} \cos ^{2} r_{b}}-\cos r_{b} \sin ^{2} r_{c}\right) .
$$

The second formula in Eq. (19) should be

$$
\mathcal{N}_{C_{I}(A B)}=\frac{1}{2}\left(-\sin ^{2} r_{c}+\sqrt{4 \cos ^{2} r_{c}+\sin ^{4} r_{c}}\right) .
$$

These amendments do not affect our conclusions; the heights of our figures are affected slightly, but all the conclusions still hold. In addition, in the Abstract, the statement "the qutrit systems are better than qubit systems" should be "the tripartite systems are better than bipartite systems."

The authors would like to thank W.-C. Qiang, G.-H. Sun, O. Camacho-Nieto, and S.-H. Dong for bringing these issues to our attention. 